Research Article

\title{
Reliability-Based Fatigue Life Prediction for Complex Structure with Time-Varying Surrogate Modeling
}

\author{
Lu-Kai Song $\mathbb{D}^{1},{ }^{1}$ Guang-Chen Bai, ${ }^{1}$ Cheng-Wei Fei $\mathbb{D}^{1},{ }^{1,2}$ and Jie Wen ${ }^{1}$ \\ ${ }^{1}$ School of Energy and Power Engineering, Beihang University, Beijing 100191, China \\ ${ }^{2}$ Department of Aeronautics and Astronautics, Fudan University, Shanghai 200433, China \\ Correspondence should be addressed to Cheng-Wei Fei; feicw544@163.com
}

Received 30 May 2018; Accepted 24 September 2018; Published 16 October 2018

Academic Editor: Pavel Lejcek

Copyright (c) 2018 Lu-Kai Song et al. This is an open access article distributed under the Creative Commons Attribution License, which permits unrestricted use, distribution, and reproduction in any medium, provided the original work is properly cited.

To improve the computational efficiency and accuracy of reliability-based fatigue life prediction for complex structure, a timevarying particle swarm optimization- (PSO-) based general regression neural network (GRNN) surrogate model (called as TV/PSO-GRNN) is developed. By integrating the proposed space-filling Latin hypercube sampling technique and PSO-GRNN regression function, the mathematical model of TV/PSO-GRNN is studied. The reliability-based fatigue life prediction framework is illustrated in respect of the TV/PSO-GRNN surrogate model. Moreover, the reliability-based fatigue life prediction of an aircraft turbine blisk under multiphysics interaction is performed to validate the TV/PSO-GRNN model. We obtain the distributional characteristics, reliability degree, and sensitivity degree of fatigue failure cycle, which are useful for the turbine blisk design. By comparing the direct simulation (FE/FV model), RSM, GRNN, PSO-GRNN, and TV/PSO-GRNN, we observe that the TV/PSOGRNN surrogate model is promising to perform the reliability-based fatigue life prediction of the turbine blisk and enhance the computational efficiency while ensuring an acceptable computational accuracy. The efforts of this study offer a useful insight for the reliability-based design optimization of complex structure.

\section{Introduction}

Complex structure possesses complex geometric modelling and endures multiple loads during operation in many mechanical systems, such as aircraft engine and spacecraft $[1-3]$. Because of significant cyclic stresses induced by fluid loads, thermal loads, and centrifugal load, as one key failure mode, the fatigue failure seriously affects the security performance of the complex structure [4]. The ever-increasing demands for high-reliability performance and low maintenance cost drive the rising attention to the life prediction approaches. To date, many efforts have quantified the fatigue life with numerical and experimental investigations via deterministic analyses, which assures the prediction security for fatigue life by relatively conservative results [5-7]. However, these efforts possess great blindness in fatigue life prediction because the randomness of various impact factors is not considered. In fact, the fatigue life shows obvious stochastic behavior in nature by multiple uncertainties, such as material properties, load fluctuations, model variabilities, and other uncontrolled stochastic variations in engineering [8-10]. Therefore, the uncertainties should be addressed directly for fatigue life prediction. One viable alternative is reliability-based fatigue life prediction approach, which does consider the uncertainties through the probabilistic-based model to predict the probability distribution of fatigue life to overcome the shortages of deterministic analysis. In view of these virtues, reliability-based fatigue life prediction has been widely used to account for the uncertainties of materials and structures, including reliability-based crack growth assessment [11-13], probabilistic strain-life fatigue modelling [14-16], probabilistic analysis for creep-fatigue behavior [17], physics of failure-based fatigue life prediction accounting for model uncertainty [18], low cycle fatigue life prediction under material variability $[19,20]$, and probabilistic life assessment using mature commercial software tools [21-24]. From the aforementioned studies, the uncertainty factors in fatigue life prediction have been 
adequately explored, and the feasibility and effectiveness of the reliability-based fatigue life prediction were validated as well. However, a few works consider multiple uncertainties in one unified reliability-based analysis regime, which neglects the combined effects among these uncertainties and there are therefore large calculating deviations on the results of fatigue life assessment $[9,25]$. Therefore, it is increasingly desired to establish unified reliability-based prediction techniques to process the uncertain factors in one uniform fatigue life prediction framework.

Under such circumstances, some reliability-based prediction techniques have emerged for the multisource uncertainty issues [26-28]. As one valuable analytical approach, Monte Carlo (MC) simulation can have high computational accuracy in reliability evaluation fields by enough samples. Owing to the requirements of performing thousands of substantial iterative calculations in analysis process [29], however, the MC simulation might incur a high computing cost in solving the nonlinear state functions of complex structure with high nonlinearity, time variation, and strong coupling. Accordingly, the MC method is generally impractical to conduct a reliability-based fatigue life prediction for complex structure. To address this issue, surrogate model is developed to avoid tremendous calculation tasks with an acceptable computational efficiency $[30,31]$. Recently, the surrogate models, such as response surface model (RSM) [32, 33], artificial neural network (ANN) [34, 35], support vector machine [36, 37], and Kriging model [38, 39], had been widely investigated in probabilistic analysis and optimization. Compared with other surrogate models, the ANN surrogate model can accomplish strong nonlinear regression in high-dimension input variables analysis and optimization problems. In this case, we propose ANN-based surrogate model to complete the reliability-based fatigue life prediction of complex structure. As an important ANN surrogate model, general regression neural network (GRNN) holds strong nonlinear mapping ability and good approximation ability, and possesses great potential to improve the computational efficiency and accuracy of reliability-based prediction [40, 41]. For complex structure, the reliability-based fatigue life prediction considering multiuncertainty variables leads to high nonlinearity, large time-varying, and strong coupling in state functions. Therefore, the traditional GRNN model still hardly satisfies the requirements of the computational efficiency and the prediction of complex structure reliabilitybased fatigue life.

To enhance the computing efficiency and accuracy of the traditional GRNN surrogate model, we first develop the PSO-GRNN surrogate model by integrating both the global searching ability of dynamic PSO algorithm and the local precise description ability of GRNN model. The dynamic PSO algorithm is a high-speed and high-accuracy optimization algorithm with great parallel computational ability $[42,43]$, which is conductive to optimize the undetermined smooth factor for the GRNN surrogate model. Therefore, the accurate smooth factor of the surrogate model can be found by adopting the PSO algorithm. However, it is difficult that the PSO-GRNN is directly applied to the nonlinear reliability-based fatigue life prediction of complex structure with time-varying characteristics. To address this issue, the time-varying PSO-GRNN (TV/PSO-GRNN) surrogate model is further proposed. Although treating time-varying fatigue life response as output random variable and generating the high-quality learning samples by the proposed space-filled Latin hypercube sampling (SLHS) technique, the mathematical regression model of TV/PSO-GRNN is established for the high-fidelity reliability-based fatigue life prediction.

The objective of this study is to present an efficient time-varying surrogate model, called as TV/PSO-GRNN, to improve the computational efficiency and accuracy of reliability-based fatigue life prediction for complex structure. As for this method, we adopt the dynamic PSO algorithm to search the smooth factor of GRNN, use the time-varying data set processing approach to treat timevarying fatigue life response as output random variable, and use the SLHS technique to generate high-quality learning samples. The feasibility and effectiveness of the proposed TV/PSO-GRNN surrogate model is verified by the reliability-based fatigue life prediction of turbine blisk in an aircraft engine with respect to multiphysics interaction.

The rest of this study is organized as follows. Section 2 discusses the basic time-varying surrogate modelling theory, including PSO-GRNN and TV/PSO-GRNN, for the reliability-based fatigue life prediction. In Section 3, the essential methodology of the reliability-based framework for fatigue life prediction with the proposed TV/PSO-GRNN is investigated. In Section 4, the deterministic analyses of fatigue life prediction are discussed. The reliability-based fatigue life prediction of turbine blisk with respect to multiphysics interaction is performed to validate the proposed TV/PSO-GRNN in Section 5. Some conclusions and outlooks on this study are summarized in Section 6.

\section{Time-Varying Surrogate Modelling Theory}

By both the global searching ability of dynamic PSO algorithm and the local precise description ability of GRNN model, the two surrogate models of both PSO-GRNN and time-varying PSO-GRNN (TV/PSO-GRNN) are developed for reliability-based fatigue life prediction. The basic architecture, space-filled sampling techniques, and mathematical model of PSO-GRNN and TV/PSO-GRNN surrogate model are discussed below.

2.1. PSO-GRNN. As an important surrogate model, general regression neural network (GRNN) is developed based on the intelligent statistical learning theory and holds high computational efficiency, good regularization ability, and strong robustness ability [41]. The complex weights training process is avoided, and the approximation ability and nonlinear mapping ability of GRNN model are only determined by one smooth factor $\sigma$. To enhance the computational accuracy of GRNN model, the PSO-GRNN surrogate model is proposed by combining the dynamic PSO 
algorithm of searching the optimal smooth factor and the GRNN model of constructing high-fidelity surrogate model. The basic thought and mathematical model of the PSOGRNN are summarized as follows.

With a certain distribution type of joint probability density function $F(\mathbf{x}, y)\left(\mathbf{x} \in \mathbf{R}^{l}, y(\mathbf{x}) \in \mathbf{R}\right)$ and the corresponding learning sample set $\left\{\left(\widehat{\mathbf{x}}_{j}, \widehat{\mathbf{y}}\right)\right\} j=1,2, \ldots, m$, to retrieve the precise output responses, the output space $\mathbf{R}$ is mapped from the input vector space $\mathbf{R}^{l}$, and the nonlinear regression function $f_{\sigma}(\mathbf{x})$ is denoted by

$$
F=\left\{f_{\sigma}(\mathbf{x}), \sigma \in \Lambda \mid f: \mathbf{R}^{l} \longrightarrow \mathbf{R}\right\},
$$

where $\Lambda$ is the data set of undetermined smooth factors $\sigma$.

In this study, the nonlinear regression function $f_{\sigma}(\mathbf{x})$ will be fitted by PSO-GRNN. The topology structure of PSOGRNN surrogate model is shown in Figure 1. As shown in Figure 1, the PSO-GRNN surrogate model refers to four different layers of input layer, pattern layer, summation layer, and output layer. Primarily, the input layer consists of the node sources of the PSO-GRNN model, and the neuron number of input layer is equal to the dimension of input random variables, then the input variables are directly transferred to pattern layer.

In the pattern layer, the total neuron number in the pattern layer is decided by the learning sample number $m$. In view of the rapid decay characteristics, the Gaussian basis function is selected as the transfer function of pattern neurons, which leads to different mapping processing units in PSO-GRNN model. In this process, multiple computation tasks simultaneously are processed to improve the fitting efficiency effectively in nonlinear mapping problems of multiple objective design. The continuous transfer function of pattern layer is expressed as

$$
P_{j}(\mathbf{x})=\exp \left[-\frac{\left(\mathbf{x}-\widehat{\mathbf{x}}_{j}\right)^{\mathrm{T}}\left(\mathbf{x}-\widehat{\mathbf{x}}_{j}\right)}{2 \sigma^{2}}\right], \quad j=1,2, \ldots, m,
$$

where $\exp (\bullet)$ denotes the exponential function operator and $\left(\mathbf{x}-\widehat{\mathbf{x}}_{j}\right)^{\mathrm{T}}\left(\mathbf{x}-\widehat{\mathbf{x}}_{j}\right)$ represents the square of Euclid distance between input vector $\mathbf{x}$ and $j$-th learning sample point $\widehat{\mathbf{x}}_{j}$.

After pattern output calculation, the summation operator will be performed in the summation layer. The arithmetical summation and weighted summation are completed in $S_{D}$ neuron and $S_{N}$ neuron, respectively. The transfer functions of $S_{D}$ neuron and $S_{N}$ neuron are defined as

$$
\left\{\begin{array}{l}
\mathbf{S}_{\mathrm{D}}=\sum_{j=1}^{m} P_{j}(\mathbf{x}), \\
\mathbf{S}_{\mathrm{N}}=\sum_{j=1}^{m} w_{j} P_{j}(\mathbf{x}),
\end{array}\right.
$$

in which $w_{j}$ indicates the connection weights of $j$-th pattern neuron and $\mathbf{S}_{\mathrm{N}}$ neuron.

Hence, the output response $y(x)$ and the nonlinear regression function $f_{\sigma}(\mathbf{x})$ can be retrieved with $\mathbf{S}_{\mathrm{N}}$ and $\mathbf{S}_{\mathrm{D}}$ :

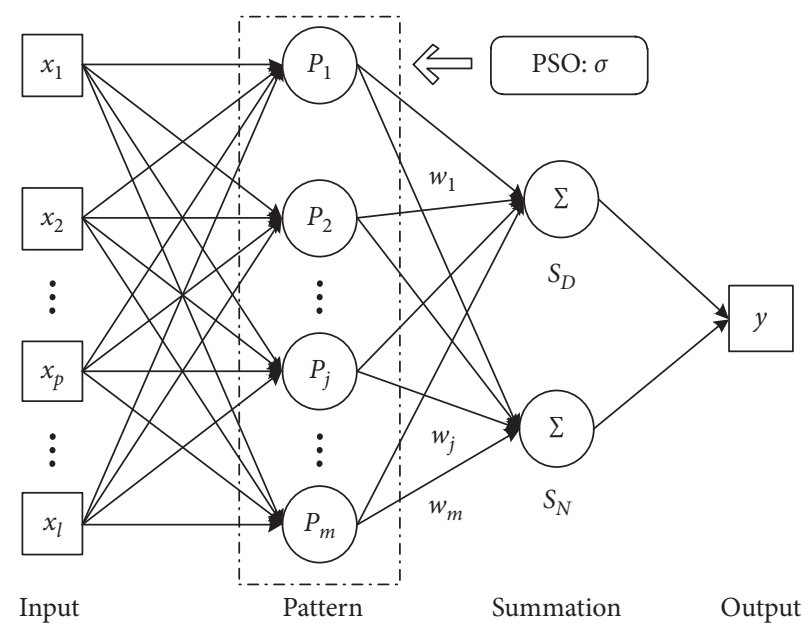

FIGURE 1: The topology structure of PSO-GRNN model.

$$
\begin{array}{r}
y(\mathbf{x})=f_{\sigma}(\mathbf{x})=\frac{\mathbf{S}_{\mathrm{N}}}{\mathbf{S}_{\mathrm{D}}}=\frac{\sum_{j=1}^{m} w_{j} \exp \left[-\left(\left(\mathbf{x}-\widehat{\mathbf{x}}_{j}\right)^{\mathrm{T}}\left(\mathbf{x}-\widehat{\mathbf{x}}_{j}\right)\right) /\left(2 \sigma^{2}\right)\right]}{\sum_{j=1}^{m} \exp \left[-\left(\left(\mathbf{x}-\widehat{\mathbf{x}}_{j}\right)^{\mathrm{T}}\left(\mathbf{x}-\widehat{\mathbf{x}}_{j}\right)\right) /\left(2 \sigma^{2}\right)\right]}, \\
j=1,2, \ldots, m .
\end{array}
$$

The feasibility and effectiveness of the aforementioned PSO-GRNN mathematical model heavily depends on the smooth factor $\sigma$. Obviously, the problem of fitting highfidelity regression function is transformed into obtaining optimal solution of the following learning model:

$$
\min \frac{1}{m} \sum_{j=1}^{m}\left[\tilde{y}\left(\widehat{\mathbf{x}}_{j}\right)-\hat{y}_{j}\right]^{2}
$$

where $\tilde{y}\left(\widehat{\mathbf{x}}_{j}\right)$ indicates the $j$-th estimated output response value and $\hat{y}_{j}$ the $j$-th real output response value.

To solve the optimization model and enhance the approximation accuracy of PSO-GRNN, the dynamic PSO algorithm is proposed in this study. PSO algorithm is a notable searching algorithm based on the collaborative searching of particle swarm, which holds advantages in searching accuracy and searching efficiency [42]. To further improve the searching efficiency and accuracy of PSO algorithm, the dynamic PSO algorithm with dynamic inertia weight and dynamic learning factors are adopted. The objective of design is to complete the dynamic searching of particle swarm and weight the relationship between global searching ability and local searching ability to acquire the better optimal solution set.

The basic thought of dynamic PSO algorithm is that the particle position is composed of smooth factors, and the fitness value adopts the learning error of PSO-GRNN model. Each particle is a potential solution for initial smooth factor of the PSO-GRNN model. In searching process, all particles search for the optimal solution in the solution space by current optimal particles and updating particle individual positions, individual extreme values and population 
extremum values. The renewal formula of particle position and velocity in dynamic PSO algorithm are determined by

$$
\begin{aligned}
& \left\{\begin{array}{l}
V_{s}^{k+1}=w_{p} V_{s}^{k}+c_{1} r_{1}\left(P_{s}^{k}-X_{s}^{k}\right)+c_{2} r_{2}\left(P_{g}^{k}-X_{s}^{k}\right), \\
X_{s}^{k+1}=X_{s}^{k}+V_{s}^{k+1},
\end{array}\right. \\
& \text { s.t. }\left\{\begin{array}{l}
w_{p}(k)=w_{p 0}-\left(w_{p 0}-w_{p K}\right) \times\left(\frac{2 k}{K}-\left(\frac{k}{K}\right)^{2}\right), \\
c_{1}(k)=c_{1 o}+\left(c_{1 K}-c_{1 o}\right) \times\left(\frac{k}{K}\right)^{2} \\
c_{2}(k)=c_{2 o}+\left(c_{2 K}-c_{2 o}\right) \times\left(\frac{k}{K}\right)^{2}
\end{array}\right.
\end{aligned}
$$

where $s$ is the $s$-th particle; $k$ the current iteration number; $K$ the maximum iteration number; $V_{s}$ the current particle velocity; $X_{s}$ the current particle position; $P_{s}$ the current individual extremum; $P_{g}$ the current population extremum; $r_{1}, r_{2}$ the random numbers during time domain $[0,1] ; w_{p}(k)$ the dynamic nonlinear inertia weight; $w_{p 0}$ the initial inertia weight; $w_{p K}$ the inertia weight at the largest number of iteration; $c_{1}(k)$ and $c_{2}(k)$ the dynamic nonlinear individual learning factor and dynamic nonlinear population learning factor in the $k$-th iteration, respectively; $c_{1 o}$ and $c_{2 o}$ the initial individual learning factor and initial population learning factor, respectively; and $c_{1 K}$ and $c_{2 K}$ the individual learning factor and population learning factor in the $K$ th iteration, respectively.

After acquiring the optimal smooth factor $\sigma$ in the learning process, the PSO-GRNN model is built. On account of the arbitrary shape property of network structure and the self-adaptation characteristics of dynamic PSO algorithm, the PSO-GRNN model can effectively reduce the approximation error and deal with the regular nonlinear optimal problem with a relatively high computing efficiency and accuracy.

2.2. TV/PSO-GRNN. For time-varying (transient or dynamic) reliability analysis and probabilistic prediction problem, the output response of each calculation loop is a random process. It is difficult to conduct the reliability analysis problem of complex structure with PSO-GRNN because of the random process of output response. Facing this situation, the conventional approaches are to establish a plenty of surrogate models in the time domain and then choose one seemingly realistic response at one time point as the computational point of reliability analysis. However, in all time loops of calculations for reliability analysis, the results calculated from one single selected computational point are not so feasible and reasonable, which leads to unacceptable computing efficiency and accuracy. To address this issue, based on the PSO-GRNN model and SLHS technique, this study develops the time-varying PSOGRNN (TV/PSO-GRNN) surrogate model to calculate an extreme response rather than a series of dynamic output responses with different input variables during the time domain $[0, T]$. This process is equivalent to transform the complicated time-varying output response process into a random output variable in each stochastic analysis. In this case, the selected random extreme response can guarantee the analytical accuracy. Obviously, the TV/PSO-GRNN is a heuristic way to improve the computational efficiency and enhance computational accuracy for reliability analysis. The analytical thought of TV/PSO-GRNN is shown in Figure 2.

In view of the basic thought of TV/PSO-GRNN in Figure 2, the extremum output response $\mathbf{Y}_{i, \max }\left(\mathbf{x}_{i}\right)$ of all dynamic response $\mathbf{Y}_{i}\left(t, \mathbf{x}_{i}\right)$ corresponding with the $i$-th input random vector $\mathbf{x}_{i}$ is obtained through a number of stochastic analyses within the time domain $[0, T]$. The data set $\left\{\mathbf{Y}_{i, \max }\left(\mathbf{x}_{i}\right): i=1,2, \ldots, n\right\}$ consisting of the maximum output responses is used to construct the TV/PSO-GRNN nonlinear regression function $f_{\sigma}(\mathbf{x})$, and the extremum response curve $y(\mathbf{x}))$ is expressed by

$$
y(\mathbf{x})=f_{\sigma}(\mathbf{x})=\left\{Y_{i, \max }\left(\mathbf{x}_{i}\right): i=1,2, \ldots, n\right\} .
$$

Clearly, the computational performance (efficiency and accuracy) of TV/PSO-GRNN surrogate model heavily depends on the feasibility and validity of data set $\mathbf{Y}_{i \text {, max }}\left(\mathbf{x}_{i}\right)$, which should be generated by high-reliability sampling technique. On this condition, the SLHS technique is introduced to generate samples points without overlap. In line with this method, the feasible and valid data set could be obtained.

Firstly, to control the data sparsity and avoid the scaling issues, all input variables $\mathbf{x}$ should be normalized into the unit cube space $\left[\mathbf{x}_{\min }^{N}, \mathbf{x}_{\max }^{N}\right]$. The normalized input variables $\mathbf{x}^{N}$ can be obtained by

$$
\mathbf{x}_{i}^{N}=\frac{\left(\mathbf{x}_{\max }^{N}-\mathbf{x}_{\min }^{N}\right)\left(\mathbf{x}_{i}-\mathbf{x}_{\min }\right)}{\left(\mathbf{x}_{\max }-\mathbf{x}_{\min }\right)}+\mathbf{x}_{\min }^{N}, \quad i=1,2, \ldots n,
$$

in which $\mathbf{x}_{i}^{N}$ indicates the $i$-th normalized input variable; $\mathbf{x}_{\min }^{N}, \mathbf{x}_{\max }^{N}$ the minimum and maximum of all normalized input variable; and $\mathbf{x}_{\min }, \mathbf{x}_{\max }$ the minimum and maximum of all input variables.

Then, split the design space into equal sized hypercubes and placing points in it, ensuring that from each occupied hypercube we could exit the design space along any direction parallel with any of the axes without encountering any other occupied hypercubes. The sampling result of SLHS technique is illustrated for three dimensions in Figure 3.

In the light of the nonlinear regression function of PSOGRNN (Equation (4)) and the generated data set, the TV/PSO-GRNN nonlinear regression function is constructed as 


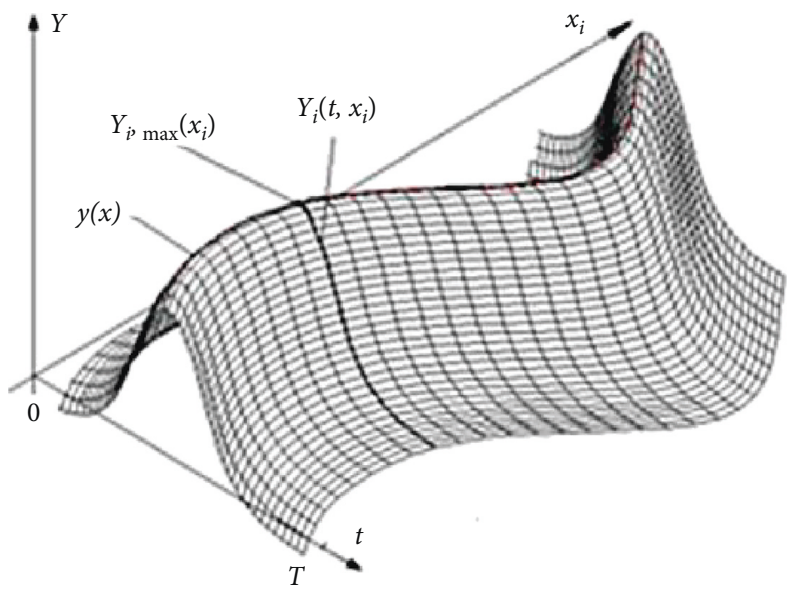

FIGURE 2: The schematic diagram of TV/PSO-GRNN.
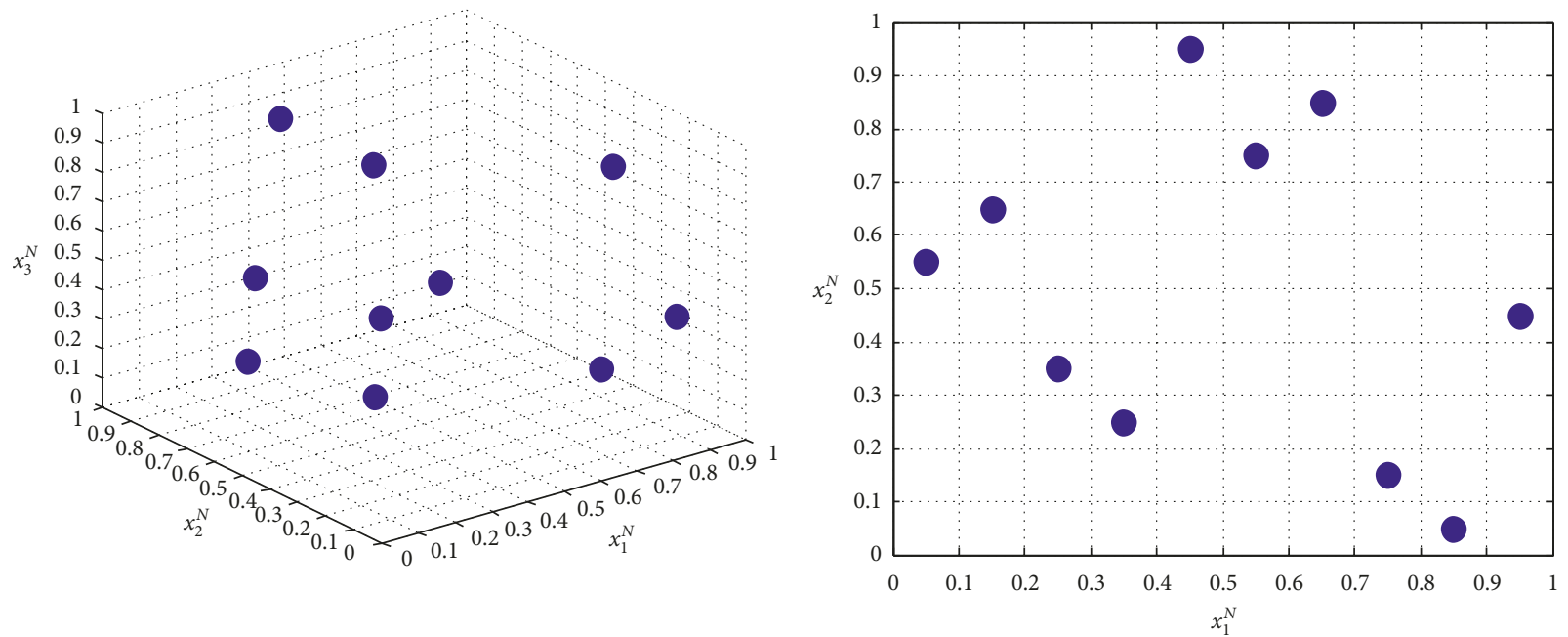

(a)

(b)

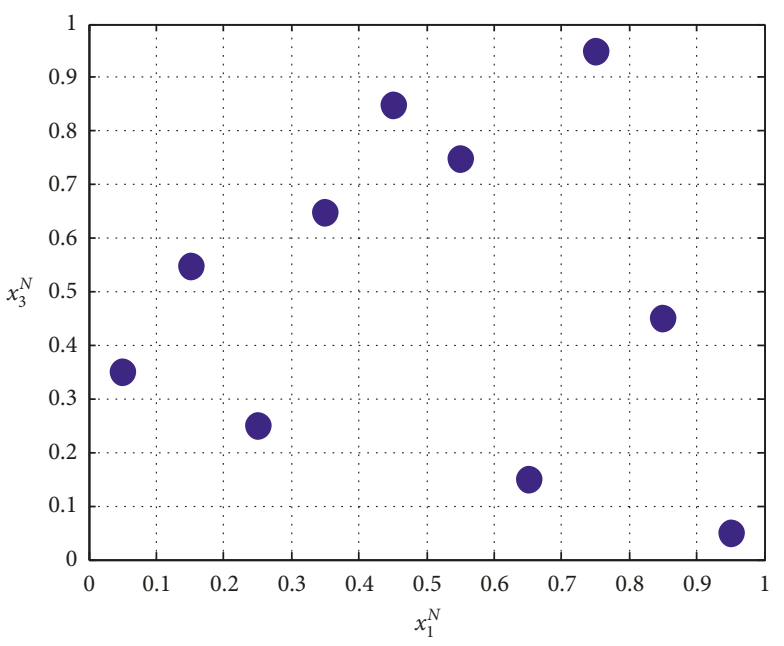

(c)

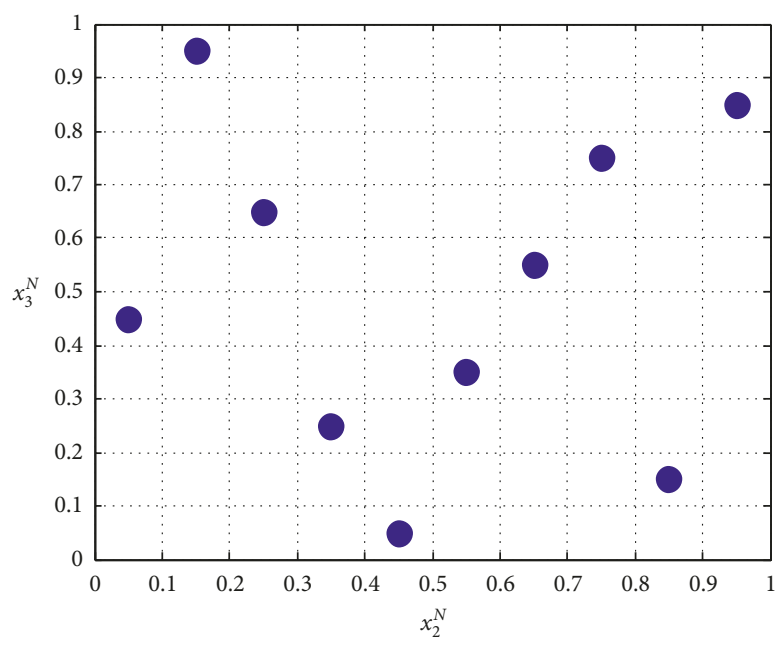

(d)

FIGURE 3: The sampling result of SLH sampling technique. (a) Three-dimension projection. (b) $\mathbf{x}_{1}^{N}-\mathbf{x}_{2}^{N}$ two-dimension projection. (c) $\mathbf{x}_{1}^{N}-\mathbf{x}_{3}^{N}$ two-dimension projection. (d) $\mathbf{x}_{2}^{N}-\mathbf{x}_{3}^{N}$ two-dimension projection. 


$$
\begin{aligned}
& Y(\mathbf{x})=\operatorname{Max}_{i} f(\mathbf{x}) \\
&=\operatorname{Max}_{i}\left\{\frac{\sum_{j=1}^{m} w_{i j} \exp \left[-\left(\left(\mathbf{x}_{i}-\widehat{\mathbf{x}}_{i j}\right)^{\mathrm{T}}\left(\mathbf{x}_{i}-\widehat{\mathbf{x}}_{i j}\right)\right) /\left(2 \sigma^{2}\right)\right]}{\sum_{j=1}^{m} \exp \left[-\left(\left(\mathbf{x}_{i}-\widehat{\mathbf{x}}_{i j}\right)^{\mathrm{T}}\left(\mathbf{x}_{i}-\widehat{\mathbf{x}}_{i j}\right)\right) /\left(2 \sigma^{2}\right)\right]}\right\}, \\
& i=1,2, \ldots, n .
\end{aligned}
$$

When the TV/PSO-GRNN nonlinear regression function is applied to evaluate the time-varying reliability of complex structure replacing the FE model, this mathematical model is called as TV/PSO-GRNN method, which is suitable to fulfill the reliability-based fatigue life prediction of complex structures.

\section{Reliability-Based Fatigue Life Prediction Framework}

In view of the elastic-plastic behavior in the fatigue life prediction of complex structure is inherently affected by the multiple stochastics of input parameters (material properties, load fluctuations, and model variabilities), deterministic analysis with the specified input variables is unsuitable to investigate the multiuncertainty features of fatigue life assessment. In this case, the reliability-based fatigue life prediction, that is, a probabilistic analysis approach, is emerged to address the multiuncertainty issues by considering the material properties, load fluctuations, and model variabilities as random variables. As for complex structure, the reliabilitybased fatigue life prediction is promising to quantify the structural reliability and determine the incidence of uncertainty parameters on the failure or reliability. In this section, the reliability-based fatigue life prediction framework with TV/PSO-GRNN model is introduced.

Assume that the allowable output structure response is $[Y]$, based on the TV/PSO-GRNN model, the limit state function $g(\mathbf{x})$ of structural fatigue life is calculated by

$$
\begin{array}{r}
g(\mathbf{x})=[Y]-\operatorname{Max}_{i}\left\{\frac{\sum_{j=1}^{m} w_{i j} \exp \left[-\left(\left(\mathbf{x}_{i}-\widehat{\mathbf{x}}_{i j}\right)^{\mathrm{T}}\left(\mathbf{x}_{i}-\widehat{\mathbf{x}}_{i j}\right)\right) /\left(2 \sigma^{2}\right)\right]}{\sum_{j=1}^{m} \exp \left[-\left(\left(\mathbf{x}_{i}-\widehat{\mathbf{x}}_{i j}\right)^{\mathrm{T}}\left(\mathbf{x}_{i}-\widehat{\mathbf{x}}_{i j}\right)\right) /\left(2 \sigma^{2}\right)\right]}\right\}, \\
i=1,2, \ldots, n
\end{array}
$$

As shown in Equation (10), $g(\mathbf{x}) \geq 0$ can ensure the safety of complex structure. With the random sampling on the established limit state function, the structural fatigue reliability degree is expressed as

$$
\begin{array}{r}
\qquad R=\frac{1}{N} \sum_{i=1}^{N} I_{r}\left[g\left(\mathbf{x}_{i}\right)\right]=\frac{N_{r}}{N}, \\
\text { s.t. } I_{r}\left[g\left(\mathbf{x}_{i}\right)\right]= \begin{cases}1, & g\left(\mathbf{x}_{i}\right) \geq 0, \\
0, & g\left(\mathbf{x}_{i}\right)<0,\end{cases}
\end{array}
$$

where $I_{r}\left[g\left(\mathbf{x}_{i}\right)\right]$ is the indicator function of the whole reliability domain; $N_{r}$ expresses the number of sample points in the secure domain; and $N$ indicates the number of the total sample points.

Accordingly, the sensitivity analysis is performed to provide insights into the influence level of the variables mean values on the failure probability. For variables with higher sensitivity, changes in their values will lead to a greater change in the failure probability, and vice versa. In particular, we compute the expected failure probability and sensitivity degree by the variable distribution characteristics from failed samples. Thus, the failure probability $P$ and the sensitivity degree $S$ of variables mean values on the failure probability are obtained by

$$
\begin{aligned}
& \left\{\begin{array}{l}
P=\frac{1}{N} \sum_{i=1}^{N} I_{f}\left[g\left(\mathbf{x}_{i}\right)\right]=\frac{N_{f}}{N}, \\
S=E\left(\frac{I_{f}\left[g\left(\mathbf{x}_{i}\right)\right]\left(\mathbf{x}_{i j}-E\left(\mathbf{x}_{j}\right)\right)}{\operatorname{Var}\left(\mathbf{x}_{j}\right)}\right),
\end{array}\right. \\
& \text { s.t. } I_{f}\left[g\left(\mathbf{x}_{i}\right)\right]=\left\{\begin{array}{l}
1, g\left(\mathbf{x}_{i}\right) \leq 0, \\
0, g\left(\mathbf{x}_{i}\right)>0,
\end{array}\right.
\end{aligned}
$$

where $P$ represents the failure probability; $S$ the sensitivity degree of the input variables effecting the output response; $I_{f}\left[g\left(\mathbf{x}_{i}\right)\right]$ is the indicator function of the whole failure domain; $N_{f}$ indicates the number of sample points in the failure domain; and $\mathbf{x}_{j}$ expresses the $j$-th input sample point. By comparing Equations (11) and (12), we can see that the failure probability $P$ complements the reliability degree $R$, as their sum must be equal to one. This is due to the fact that a sample can only be deemed as a success or a failure, but not "none" or "both."

To improve the computational efficiency and accuracy of the reliability-based fatigue life prediction of complex structure, the reliability-based fatigue life prediction framework is constructed based on the time-varying powerful mapping ability of TV/PSO-GRNN surrogate model, which is illustrated in Figure 4.

\section{Deterministic Analysis with Multiphysics Interaction}

As a vital component of an aircraft engine, the high-pressure turbine blisk shown in Figure 5 endures multidisciplinary loads (gas pressure loads, heat loads, and centrifugal force) from multiple physical fields (fluid field, thermal field, and structural field). The multidisciplinary loads are timevarying and strong coupling in operating process, so that it is easy to lead to tensile stresses and serious low cycle fatigue damage. The load spectrum of an aircraft engine is shown in Figure 6 [44]. Furthermore, the specific values of nonlinear material parameters, including elastic modulus $E$, heat transfer coefficient $\lambda$, expansion coefficient $\alpha$, and 




FIgURE 4: The essential process with TV/PSO-GRNN surrogate model.



Figure 5: The schematic diagram of an aeroengine turbine blisk. 


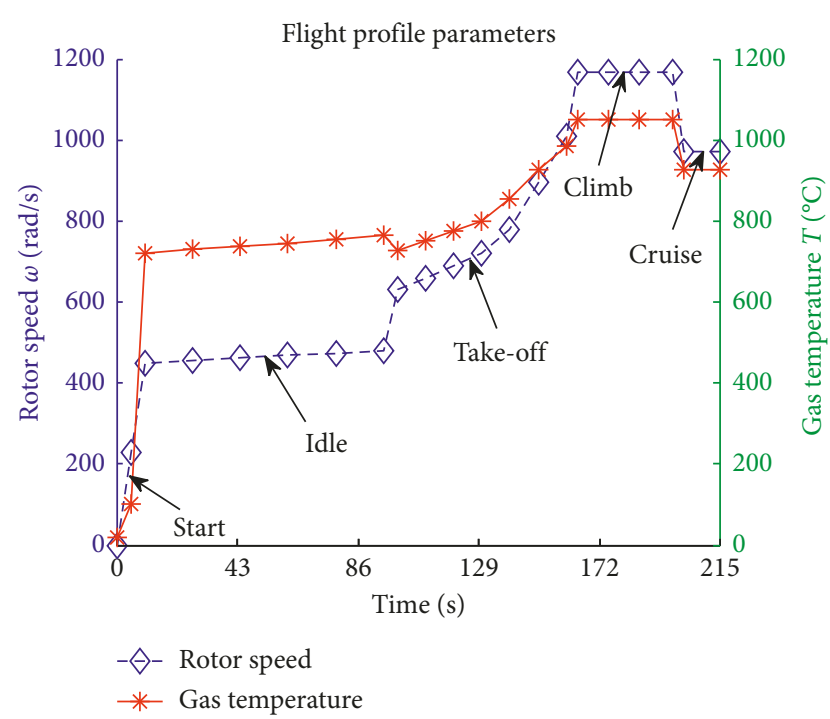

FIGURE 6: The loads spectrum of an aircraft engine.

Poisson's ratio $\mu$, are varying with temperature, and its detailed nonlinear variation characteristics are shown in Table 1.

To reasonably assess the reliability and performance of turbine components and whole aircraft engine, it is necessary to predict the fatigue life of turbine blisk. Therefore, the low cycle fatigue life prediction of an aeroengine turbine blisk is regarded as the objective of study. The FE model of the turbine blisk is drawn in Figure 7.

To simplify the simulation complexity and cut the computational task, we decompose the multidisciplinary coupling system into several simple single-disciplinary subsystems (fluid subsystem, thermal subsystem, and structure subsystem). Each subsystem is assumed to be independent mutually in operation. Besides, the loads and responses are transferred among subsystems by multiphysics interaction (MPI) surface. The MPI sketch of turbine blisk is drawn in Figure 8. In fluid subsystem, the related parameters are $168 \mathrm{~m} / \mathrm{s}$ for inlet fluid velocity, $600,000 \mathrm{~Pa}$ for inlet pressure, $11756 \mathrm{~W} / \mathrm{m}^{2} \mathrm{~K}$ for heat transfer coefficient in MPI surface, and $1 \mathrm{~atm}$ for outlet pressure. During the fluid dynamics analysis, the pressure distribution on MPI surface is obtained in Figure 9. After acquiring the initial temperature loads from fluid subsystem, we select the related thermal parameters of $21.2 \mathrm{~W} /\left(\mathrm{m}^{\circ} \mathrm{C}\right)$ for thermal conductivity coefficient and $14.8 \times 10^{-6 \circ} \mathrm{C}^{-1}$ for thermal expansion coefficient. According to the temperature consistency principle, the thermal analysis is completed to gain the body temperature distribution on the MPI surface in Figure 10. The fluid pressure distribution loads and temperature distribution loads are transmitted to the structure subsystem. And we select material density $8.24 \mathrm{~g} / \mathrm{cm}^{3}$, elastic modulus $160 \mathrm{GPa}$, and the flight profile parameters in Figure 4. Thereby, the deterministic analysis of turbine blisk is performed with MPI, in which the maximum stress distribution and strain range distribution are shown in Figure 11.

As illustrated in the analytical results, the maximum stress $\sigma_{\max }$ and strain range $\Delta \varepsilon_{t}$ of turbine blisk reach at the
TABle 1: The variation characteristics of three nonlinear parameters.

\begin{tabular}{lccccccccc}
\hline $\begin{array}{l}\text { Temperature } \\
\left({ }^{\circ} \mathrm{C}\right)\end{array}$ & 100 & 200 & 300 & 400 & 500 & 600 & 700 & 800 & 900 \\
\hline$E(\mathrm{MPa})$ & 204 & 203 & 192 & 181 & 176 & 160 & 150 & 141 & 136 \\
$\lambda\left(\mathrm{W} /\left(\mathrm{m}^{\circ} \mathrm{C}\right)\right)$ & 14.7 & 15.9 & 17.8 & 18.3 & 19.6 & 21.2 & 22.8 & 23.6 & 27.6 \\
$\alpha\left(10^{-6 \circ} \mathrm{C}\right)$ & 11.8 & 13 & 13.5 & 14.1 & 14.4 & 14.8 & 15.4 & 17 & 18.4 \\
$\mu(\mathrm{m} / \mathrm{m})$ & 0.3 & 0.3 & 0.3 & 0.31 & 0.31 & 0.32 & 0.32 & 0.33 & 0.33 \\
\hline
\end{tabular}

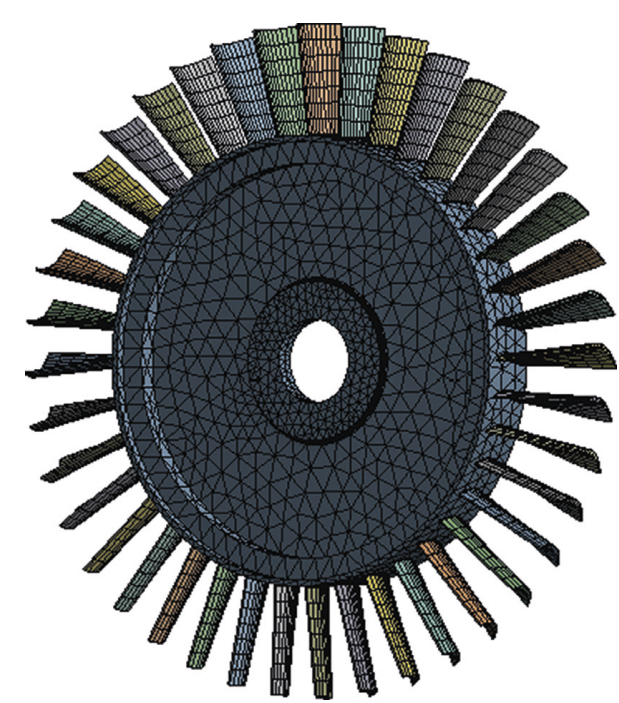

FIgURE 7: The FE model of turbine blisk.



FIgURE 8: The MPI sketch of turbine blisk

peak values at the back of blade root. Considering the main stress cycle $0-\sigma_{\max }-0$, the fatigue life can be obtained as 3228 flight cycles by the improved Masson-Coffin model [29]:

$$
\frac{\Delta \varepsilon_{\mathrm{t}}}{2}=\frac{\sigma_{\mathrm{f}}^{\prime}-\sigma_{\mathrm{m}}}{E}\left(2 N_{\mathrm{f}}\right)^{b}+\varepsilon_{\mathrm{f}}^{\prime}\left(2 N_{\mathrm{f}}\right)^{c},
$$

where $\sigma_{\mathrm{m}}$ represents the mean stress; $\sigma_{\mathrm{f}}^{\prime}$ the fatigue strength coefficient; $\varepsilon_{\mathrm{f}}^{\prime}$ the fatigue ductility coefficient; $b$ the fatigue strength exponent; $c$ the fatigue ductility exponent; and $N_{\mathrm{f}}$ the failure cycle number. 


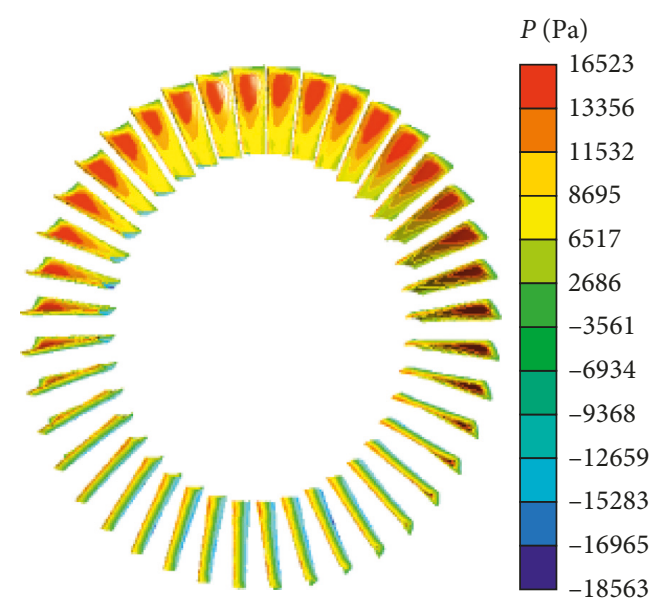

FIgURE 9: The pressure distribution on turbine blisk surface.

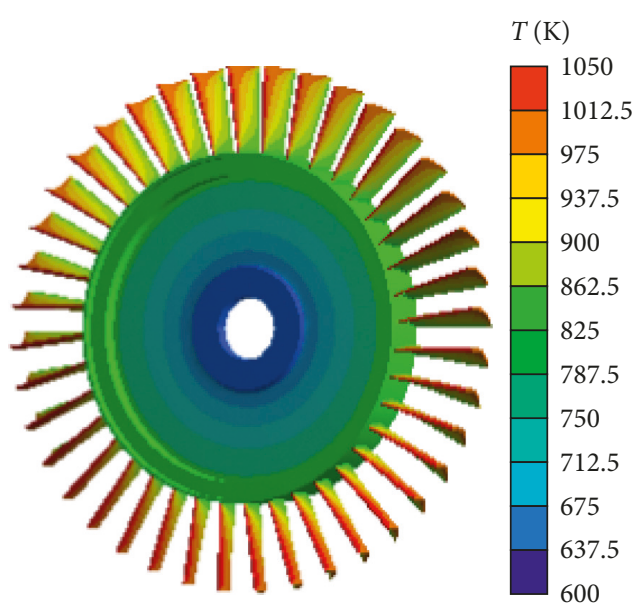

Figure 10: The temperature distribution on turbine blisk surface.

\section{Transient Reliability-Based Fatigue Life Prediction}

In this section, the transient reliability-based fatigue life prediction of turbine blisk is performed with the proposed TV/PSO-GRNN model, by considering the nonlinearities of material properties and multiphysics loads in operation. All computations are performed on an Inter(R) Core(TM) Desktop Computer (3 GHz CPU and $16 \mathrm{~GB}$ RAM).

5.1. Random Variables Selection. Under aircraft engine operation, the material properties, multiphysical loads, and model uncertainty possess evidently inherent randomness and seriously influence the fatigue life of turbine blisk $[45,46]$. Therefore, we regarded rotor speed $\omega$, gas temperature $T$, fluid velocity $\rho$, elastic modulus $E$, and thermal conductivity coefficient $\lambda$ as random variables. The distribution characteristics of physical variables are listed in Table 2. The distribution characteristics of model uncertainty parameters such as the fatigue strength exponent $b$, fatigue ductility exponent $c$, fatigue strength coefficient $\sigma_{\mathrm{f}}^{\prime}$, and fatigue ductility coefficient $\varepsilon^{\prime}{ }_{\mathrm{f}}$ are also considered as random variables as listed in Table 3 [47]. Assuming that the fatigue ductility coefficient $\varepsilon_{\mathrm{f}}^{\prime}$ obeys lognormal distribution and other random variables obey normal distribution, and all of selected random variables are reciprocally independent, respectively.

5.2. TV/PSO-GRNNM Surrogate Modelling. The uncertainty parameters in Tables 2 and 3 are regarded as the input variables, and the failure cycle number $N_{\mathrm{f}}$ of turbine blisk is taken as the output response. In light of Latin hypercube sampling and FE simulations, 20 groups of training samples and 100 groups of test samples are extracted. The TV/PSOGRNN surrogate model is constructed by training the surrogate model. The test result of TV/PSO-GRNN surrogate model is drawn in Figure 12. To quantify approximation error and evaluate the fitting performance, the mean relative error (MRE) metric and root mean squared error (RMSE) matrix in Equation (14) are adopted to reveal. The comparison results with response surface model (RSM) [33], GRNN, PSO-GRNN, and TV/PSO-GRNN surrogate model are shown in Table 4:

$$
\left\{\begin{array}{l}
\operatorname{MRE}=\frac{1}{n_{t}} \sum_{i=1}^{n_{t}}\left|\left(y_{i}-\tilde{y}_{i}\right) / y_{i}\right|, \\
\operatorname{RMSE}=\sqrt{\left(\sum_{i=1}^{n_{t}}\left(y_{i}-\tilde{y}_{i}\right)\right)^{2} / n_{t}},
\end{array}\right.
$$

where $\mathbf{y}$ is the real output response; $\tilde{\mathbf{y}}$ estimated output response; $i$-th test sample point; and $n_{t}=100$ number of test samples.

As shown in Figure 10 and Table 4, even if the fatigue life of turbine blisk possesses large dispersion, the TV/PSOGRNN surrogate model still fit each test points with almost zero approximation errors. Thus, the approximation performance of TV/PSO-GRNN model is superior to RSM, GRNN, and PSO-GRNN. Therefore, as surrogate model the TV/PSO-GRNN model is suitable to fulfill the reliabilitybased fatigue life prediction.

5.3. Reliability-Based Fatigue Life Prediction. In view of the distribution types listed in Tables 1 and 2 and MC simulation, 10,000 groups of input variables samples are obtained. The detailed probabilistic distribution characteristics are revealed in Figure 13. According to the extracted input variables and TV/PSO-GRNN model, 10,000 simulations are executed to predict the failure cycle number $N_{\mathrm{f}}$ of turbine blisk. The simulation history and probabilistic distribution of fatigue life are revealed in Figure 14, which indicates that the output response $N_{\mathrm{f}}$ of turbine blisk obeys a lognormal distribution and the fatigue life of turbine blisk under reliability $99.87 \%$ is 3265 cycles.

Through the sensitivity analysis based on Equation (12), the sensitivities and effect probability of input variables on turbine blisk fatigue failure are revealed in Figure 15. The analysis results explain that rotor speed $w$ is the most 


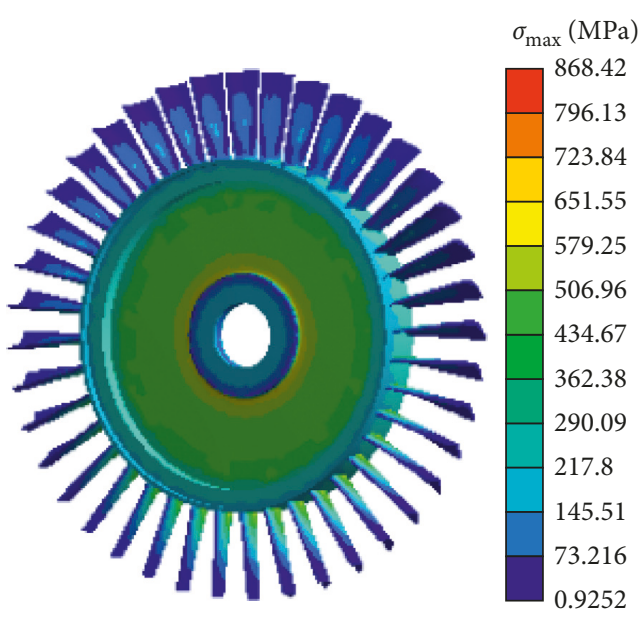

(a)

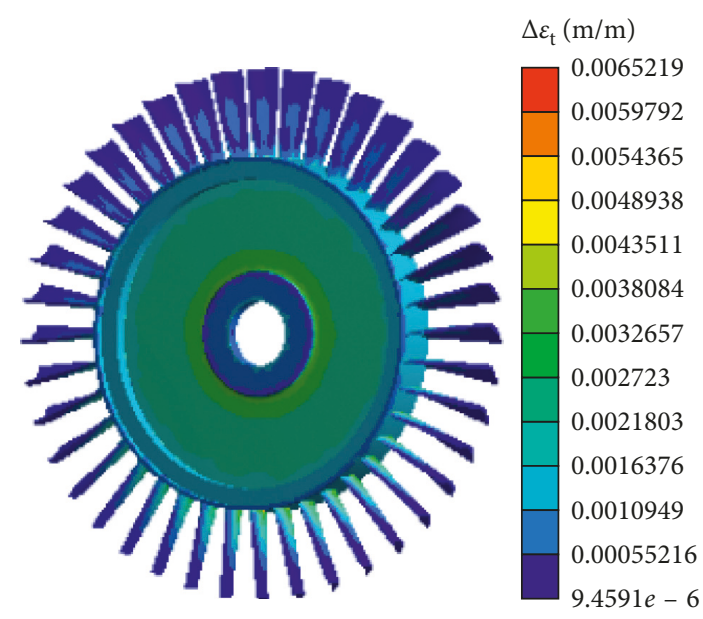

(b)

FIgURE 11: The maximum stress (a) and strain range (b) distributions on turbine blisk.

TABle 2: The distribution characteristics of physical variables.

\begin{tabular}{lccccc}
\hline Variables & $\begin{array}{c}\omega \\
(\mathrm{rad} / \mathrm{s})\end{array}$ & $T\left({ }^{\circ} \mathrm{C}\right)$ & $v(\mathrm{~m} / \mathrm{s})$ & $\begin{array}{c}E \\
(\mathrm{GPa})\end{array}$ & $\begin{array}{c}\lambda \\
\left(\mathrm{W} /\left(\mathrm{m}^{\circ} \mathrm{C}\right)\right)\end{array}$ \\
\hline Mean & 1168 & 1050 & 168 & 160 & 21.2 \\
Variance & 23.36 & 21 & 3.36 & 3.2 & 0.424 \\
Distribution & Normal & Normal & Normal & Normal & Normal \\
\hline
\end{tabular}

TABLE 3: The distribution characteristics of model variables.

\begin{tabular}{lcccc}
\hline Variables & $b$ & $c$ & $\sigma_{\mathrm{f}}^{\prime}$ & $\varepsilon_{\mathrm{f}}^{\prime}$ \\
\hline Mean & -0.09 & -0.58 & 1476 & 0.108 \\
Variance & 0.0018 & 0.0116 & 29.52 & 0.0022 \\
Distribution & Normal & Normal & Normal & Lognormal \\
\hline
\end{tabular}

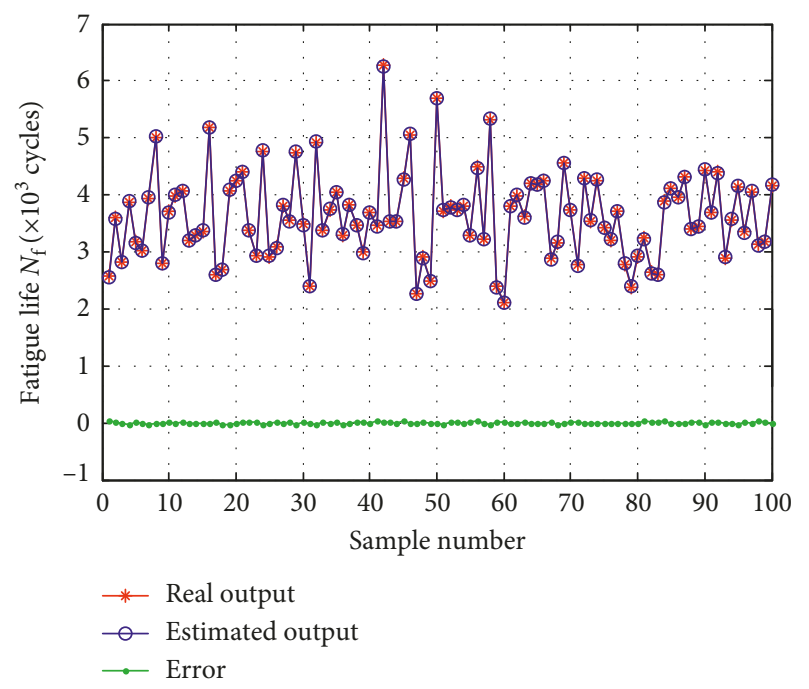

FIGURE 12: The prediction results of collaborative WNNR.

important factors and plays a leading role for the fatigue life failure with the effect probability of $32 \%$. Besides, gas temperature $T$ and fatigue strength coefficient $\sigma_{\mathrm{f}}^{\prime}$ are also
TABLE 4: The approximation performance with different surrogate models.

\begin{tabular}{lcccc}
\hline \multirow{2}{*}{ Metrics } & \multicolumn{4}{c}{ Surrogate models } \\
& RSM & GRNN & PSO-GRNN & TV/PSO-GRNN \\
\hline MRE & 1.5537 & 0.5691 & 0.4145 & 0.1456 \\
RMSE & 0.1275 & 0.1154 & 0.0663 & 0.0263 \\
\hline
\end{tabular}

main factors with the effect probabilities of $25 \%$ and $21 \%$, respectively, while the influences of other factors are weak relatively. In the fatigue failure design of turbine blisk, the rotor speed $w$, gas temperature $T$, and fatigue strength coefficient $\sigma_{\mathrm{f}}^{\prime}$ should be controlled preferentially. The fluid velocity $v$ and thermal conductivity coefficient $\lambda$ are negatively correlated with the fatigue failure probability. The other variables are positively correlated with the fatigue failure probability, which basically agree with engineering practice.

5.4. TV/PSO-GRNN Method Validation. To support the proposed TV/PSO-GRNN model, the reliability-based fatigue life prediction of the blisk is performed with $\mathrm{MC}$ method, RSM, GRNN, and PSO-GRNN, respectively. The simulation consumptions with different methods are listed in Table 5. To validate the feasibility and effectiveness of the proposed TV/PSO-GRNN model, the uniform computational efficiency (UCE) and uniform computational accuracy (UCA) are introduced in Equation (15). The comparison results of the four methods under different simulations are listed in Table 6:

$$
\left\{\begin{array}{l}
\mathrm{UCE}=\left|\frac{\widetilde{\mathrm{FT}}+\widetilde{\mathrm{CT}}-\overline{\mathrm{CT}} \mid \times 100 \%,}{\overline{\mathrm{CT}}}\right| \\
\mathrm{UCA}=\left(\frac{\operatorname{cov}\left(\overline{N_{\mathrm{f}}}, \tilde{N_{\mathrm{f}}}\right)}{\sqrt{\operatorname{var}\left(\overline{N_{\mathrm{f}}}\right) \operatorname{var}\left(\widetilde{N_{\mathrm{f}}}\right)}}\right)^{2} \times 100 \%,
\end{array}\right.
$$




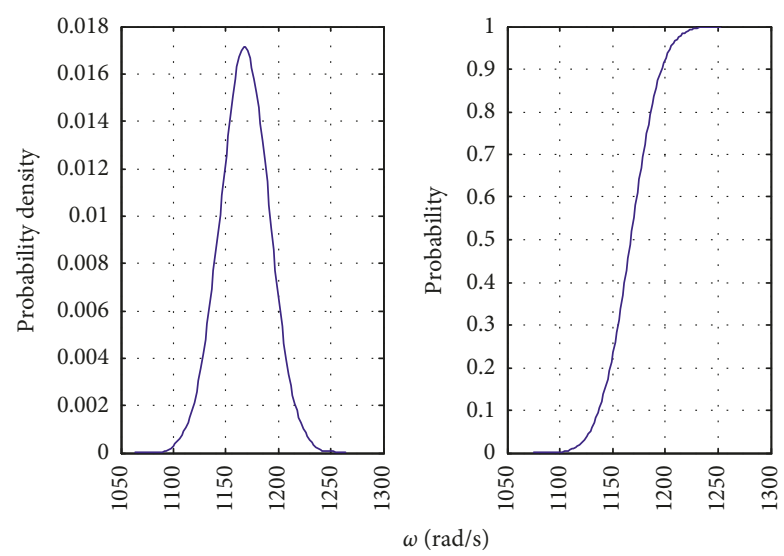

(a)


(c)

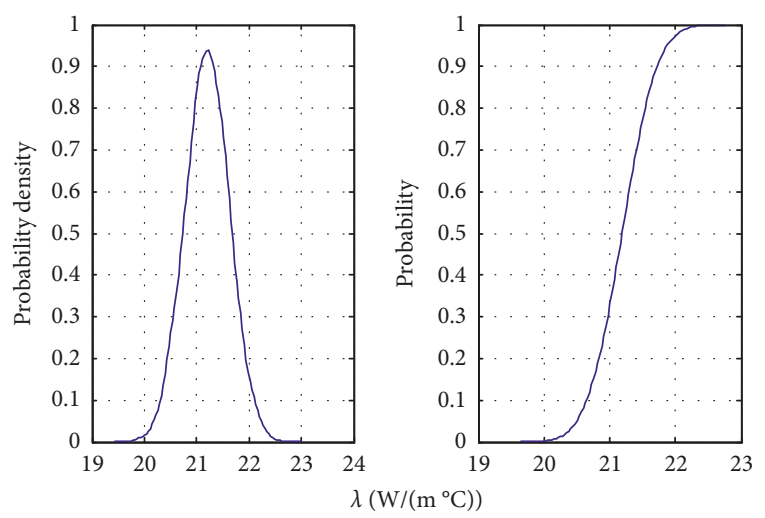

(e)
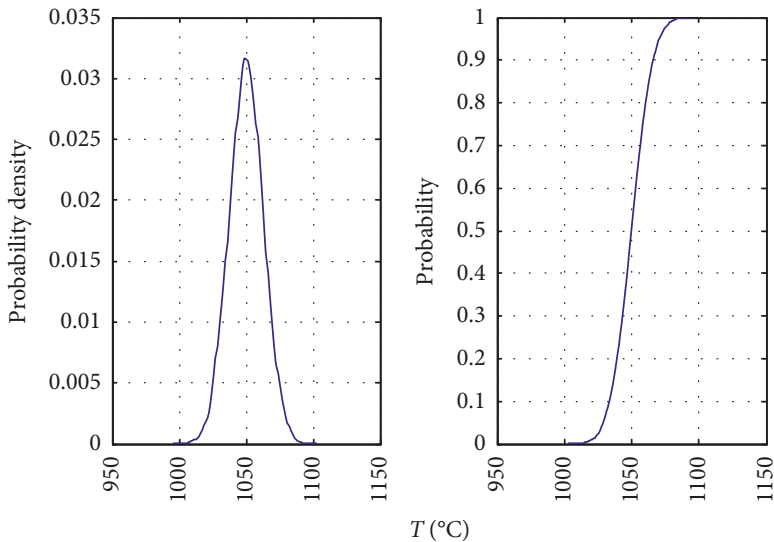

(b)



(d)
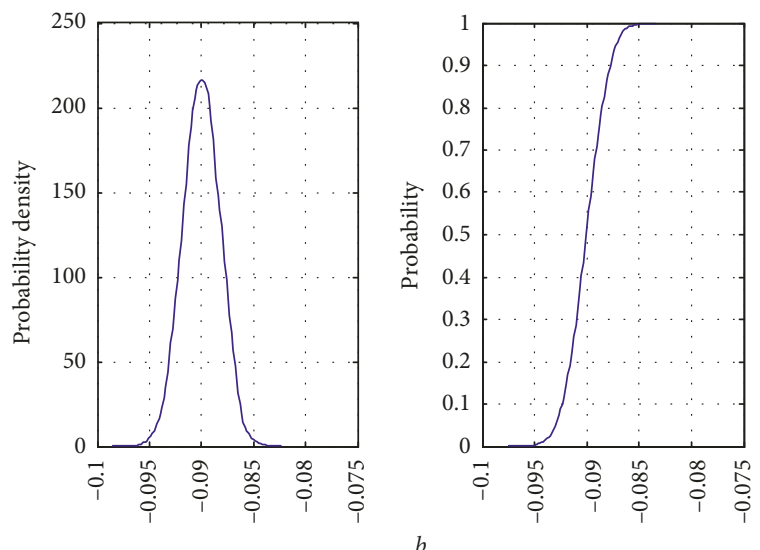

(f)

FIgURE 13: Continued. 

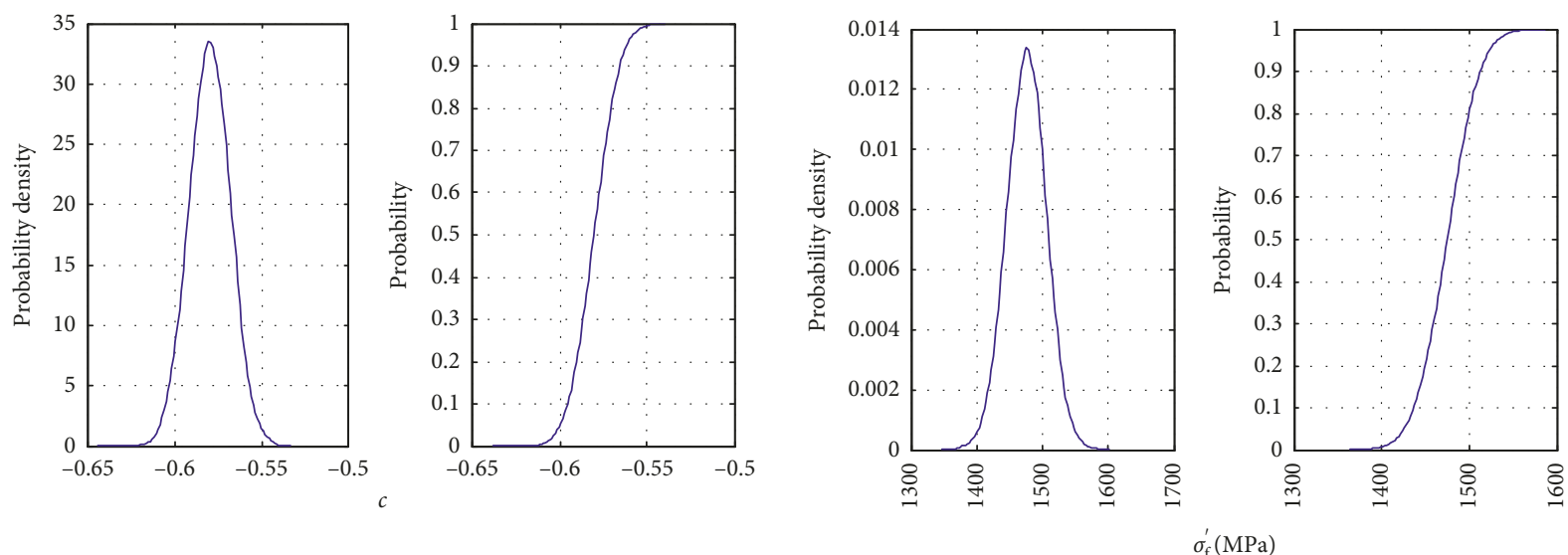

(g)

(h)


(i)

FIGURE 13: The probability distribution of input variables: (a) rotor speed, (b) gas temperature, (c) fluid velocity, (d) elastic modulus, (e) thermal conductivity coefficient, (f) fatigue strength exponent, (g) fatigue ductility exponent, (h) fatigue strength coefficient, and (i) fatigue ductility coefficient.

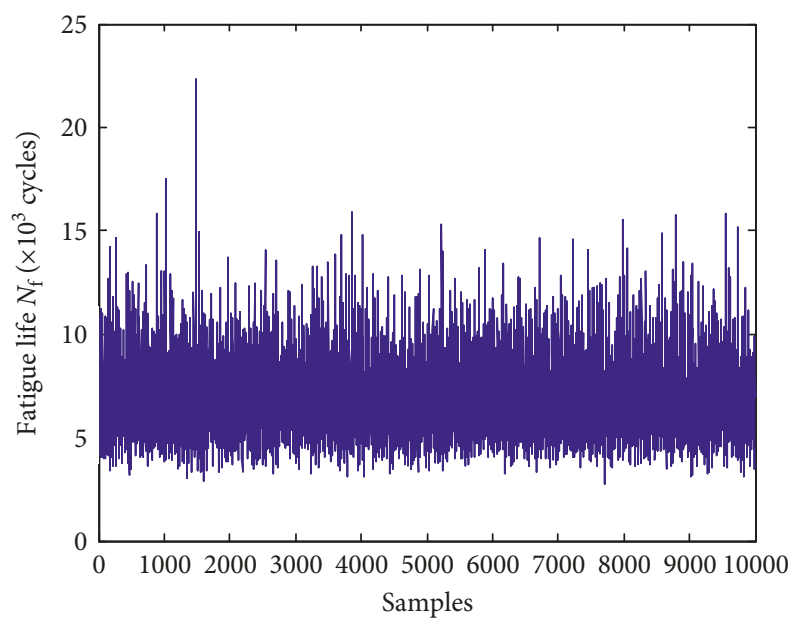

(a)

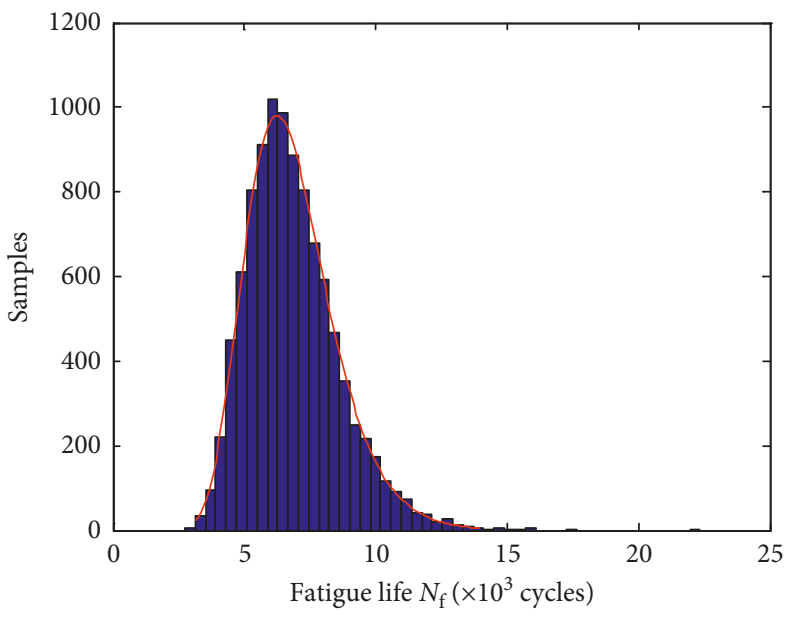

(b)

FIGURE 14: The fatigue life of turbine blisk: (a) simulation history and (b) histogram distribution. 

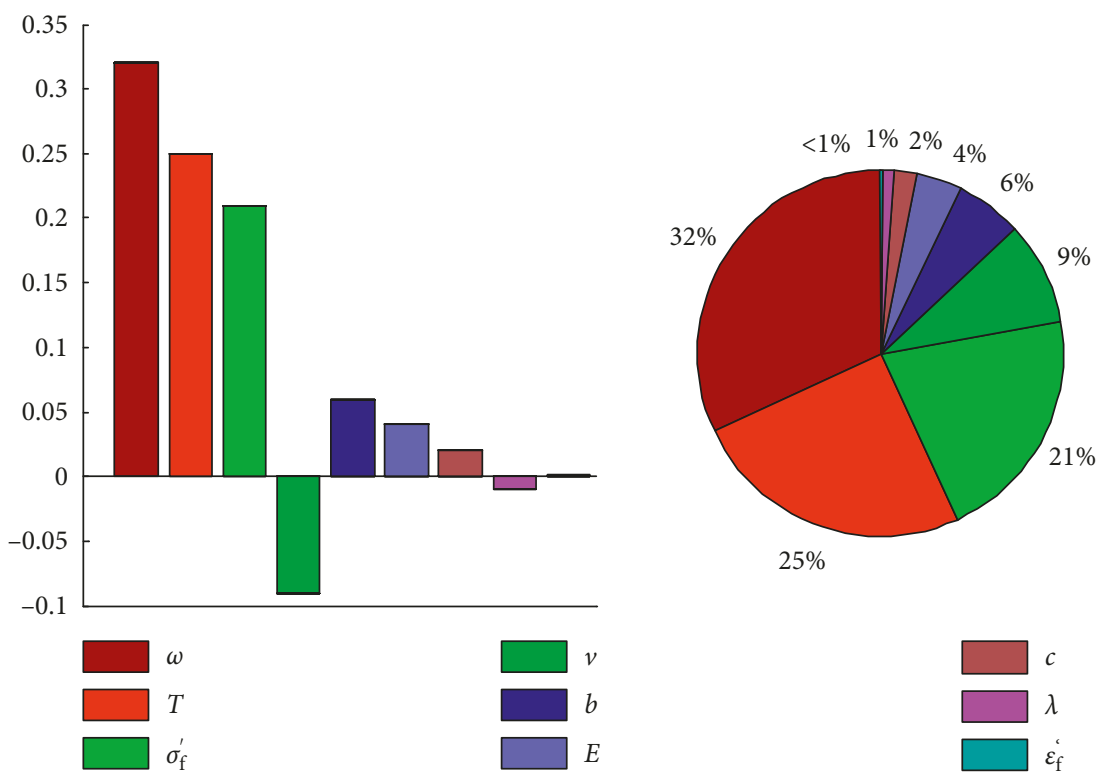

FIgURE 15: The sensitivities and effect probabilities of fatigue life.

TABLE 5: The simulation time of the reliability-based fatigue life prediction of turbine blisk.

\begin{tabular}{lccccc}
\hline \multirow{2}{*}{ Methods } & \multicolumn{2}{c}{ Fitting surrogate models } & \multicolumn{3}{c}{ Computing time of different simulation times, s } \\
& Sample number & Fitting time (s) & $10^{2}$ & $10^{3}$ & $10^{4}$ \\
\hline Direct simulation & - & - & $8.79 \times 10^{3}$ & $7.65 \times 10^{4}$ & $7.88 \times 10^{5}$ \\
RSM & 166 & $8.14 \times 10^{3}$ & 5.68 & 11.43 & 102.75 \\
GRNN & 115 & $6.73 \times 10^{3}$ & 2.42 & 6.53 & 897.13 \\
PSO-GRNN & 88 & $4.92 \times 10^{3}$ & 1.72 & 3.75 & 77.47 \\
TV/PSO-GRNN & 50 & $3.56 \times 10^{3}$ & 0.72 & 0.94 & 11.42 \\
\hline
\end{tabular}

TABLE 6: Uniform computational efficiency and accuracy of four surrogate models.

\begin{tabular}{lcccccccc}
\hline \multirow{2}{*}{ Simulation } & \multicolumn{3}{c}{ Uniform computational efficiency (UCE) (\%) } & \multicolumn{3}{c}{ Uniform computational accuracy (UCA) (\%) } \\
& RSM & GRNN & PSO-GRNN & TV/PSO-GRNN & RSM & GRNN & PSO-GRNN & TV/PSO-GRNN \\
\hline $10^{2}$ & 7.32 & 23.41 & 44.01 & 59.49 & 90.38 & 93.96 & 97.91 & 99.58 \\
$10^{3}$ & 89.34 & 91.19 & 93.56 & 95.34 & 90.92 & 94.66 & 98.52 & 99.72 \\
$10^{4}$ & 98.95 & 99.14 & 99.37 & 99.55 & 91.76 & 95.15 & 98.92 & 99.85 \\
\hline
\end{tabular}

where $\widetilde{\mathrm{FT}}$ and $\widetilde{\mathrm{CT}}$ are fitting time and computing time for surrogate models, respectively; $\overline{\mathrm{CT}}$ the time of $\mathrm{MC}$ simulations; and $\widetilde{N_{\mathrm{f}}}$ and $\overline{N_{\mathrm{f}}}$ the failure cycle number obtained by surrogate models and direct simulation (FE/FV), with $\mathrm{MC}$ method, respectively.

5.5. Discussion. As depicted in Figure 14, the fatigue life $N_{\mathrm{f}}$ of turbine blisk obeys a log-normal distribution and the fatigue life of turbine blisk under the reliability $99.87 \%$ is 3265 cycles. As revealed in Figure 15, the rotor speed $w$, gas temperature $T$, and fatigue strength coefficient $\sigma_{\mathrm{f}}^{\prime}$ are the leading factors on the fatigue life of turbine blisk as the effect probabilities are $32 \%, 25 \%$, and $21 \%$, respectively. The influences from other factors are relatively weak. Therefore, $\omega$ and $T$ should be considered with the priority in the reliability-based fatigue life design of turbine blisk. The reduction of inlet gas velocity $v$ and fatigue ductility coefficient $\varepsilon_{\mathrm{f}}^{\prime}$ causes the increase of blisk failure probability, while the increase of other parameters leads to the increase of blisk failure probability.

As revealed in Table 4 and Table 5, the fitting time and fitting number of TV/PSO-GRNN are less than RSM, GRNN, and PSO-GRNN, and the simulation consumption and total computational efficiency of TV/PSO-GRNN is superior than RSM, GRNN, PSO-GRNN, and direct simulation model as well. Moreover, the computational efficiency benefits of the proposed TV/PSO-GRNN are more obvious with increasing simulations. Hence, the proposed TV/PSO-GRNN holds high computing efficiency due to low time consumption. The main reasons are (1) TV/PSOGRNN only focus on the extremum value of the response 
process for each calculation within a time domain in the reliability-based fatigue life prediction, rather than the whole dynamic response process; (2) the TV/PSO-GRNN model can rapidly fit the nonlinear regression functions because the GRNN reduces the network complexity of surrogate model, and the dynamic PSO algorithm effectively avoid the blind searching and poor initial training values in learning process by searching optimal smooth factor with the proposed dynamic inertia weight and dynamic learning factors; (3) SLHS technique also brings more effective learning data sets for establishing satisfactory surrogate model to avoid numerous loop iterations in learning process. Therefore, based on the aforementioned strengths, the optimal smooth factor of TV/PSO-GRNN are quickly obtained, which save time and improve computing efficiency in reliability-based fatigue life prediction. Therefore, the TV/PSO-GRNN model possesses high computational efficiency in reliability-based fatigue life prediction of complex structure.

As unveiled in Figure 12 and Table 4, the established TV/PSO-GRNN surrogate model can approximate every test sample so that the proposed TV/PSO-GRNN possesses the lowest fitting error. As illustrated in Table 6, the TV/PSOGRNN is more precise than the RSM, GRNN, and PSOGRNN and is almost consistent with the direct simulation (FE/FV) method. The TV/PSO-GRNN thus holds good generalization ability and high-accuracy computational ability. The reasons are listed as follows: (1) the TV/PSOGRNN regression function is fitted based on GRNN with strong nonlinear mapping ability, (2) the global optimal smooth factor is obtained rather than local optimal solution by the dynamic PSO algorithm balance ability of global searching and local searching to ensure the computing accuracy, and (3) SLHS technique generates high-quality learning data set with less data noise, which also provides a good way to enhance the fitting ability of surrogate model. Therefore, the TV/PSO-GRNN model holds high computational accuracy in reliability-based fatigue life prediction of complex structure.

In summary, the proposed TV/PSO-GRNN greatly saves computing time and improves computational efficiency while keeping computational accuracy. Therefore, the TV/PSOGRNN is a feasible and effective way in the reliabilitybased fatigue life prediction of complex structures.

\section{Conclusions and Outlooks}

To improve the computational efficiency and accuracy of reliability-based fatigue life prediction for complex structure, an efficient time-varying surrogate model (termed “TV/PSO-GRNN") is developed. The reliability-based fatigue life prediction of turbine blisk was taken as a case to support the validation and feasibility of TV/PSO-GRNN. Some conclusions are drawn as follows:

(1) Through the reliability-based fatigue life prediction of turbine blisk with the TV/PSO-GRNN, the simulation histories, and distribution features of reliability-based fatigue life are obtained. Moreover, the high sensitivity parameters (i.e., rotor speed, gas temperature, and fatigue strength coefficient) are also retrieved, which provides a valuable reference for the design and optimization of turbine blisk.

(2) By comparing several methods (i.e., direct simulation, RSM, GRNN, and PSO-GRNN), the developed TV/PSO-GRNN possesses the highest computational efficiency and accuracy. We also demonstrated that the TV/PSO-GRNN surrogate model is an effective approach for the reliability-based fatigue life prediction of complex structure.

(3) The efforts of this study enrich mechanical reliability theory from a surrogate modelling perspective, and shed light on the further applications in the reliability-based design optimization as well.

Although this investigation provides a novel time-varying surrogate model to improve the modelling accuracy and simulation efficiency for the reliability-based fatigue life prediction of complex structure, there are still some limitations that need to be addressed in future. Most deviations from the expected solution are likely to be attributed to inaccurate information caused by the parameters of reliabilitybased fatigue life prediction framework. To further develop the fatigue life prediction of complex structure, more reasonable analysis and design techniques should be developed. Moreover, advanced time-varying regression functions based on different surrogate models should be established in future to accomplish the reliability-based design optimization for fatigue life prediction of complex structure.

\section{Acronyms}

ANN:

GRNN: General regression neural network

MPI: Multiphysics interaction

MRE: $\quad$ Mean relative error

PSO: $\quad$ Particle swarm optimization

PSO-GRNN: PSO-based GRNN

RMSE: $\quad$ Root mean squared error

RSM: Response surface model

SLHS: $\quad$ Space-filled Latin hypercube sampling

TV/PSO-GRNN: Time-varying PSO-GRNN

UCA: Uniform computational accuracy

UCE: Uniform computational efficiency.

\section{Data Availability}

The data used to support the findings of this study are available from the corresponding author upon request.

\section{Conflicts of Interest}

The authors declare that there are no conflicts of interests regarding the publication of this article.

\section{Acknowledgments}

This paper was co-supported by the National Natural Science Foundation of China (Grant nos. 51475026, 51335003, and 51605016). The authors would like to thank them. 


\section{References}

[1] S. Beretta, S. Foletti, M. Madia, and E. Cavalleri, "Structural integrity assessment of turbine discs in presence of potential defects: probabilistic analysis and implementation," Fatigue \& Fracture of Engineering Materials \& Structures, vol. 38, no. 9, pp. 1042-1055, 2015.

[2] L. K. Song, C. W. Fei, J. Wen, and G. C. Bai, "Multi-objective reliability-based design optimization approach of complex structure with multi-failure modes," Aerospace Science and Technology, vol. 64, pp. 52-62, 2017.

[3] C. Y. Zhang, C. Lu, C. W. Fei, L. J. Liu, Y. S. Choy, and X. G. Su, "Multi-object reliability analysis of turbine blisk with multi-discipline under multi-physical field interaction," $A d$ vances in Materials Science and Engineering, vol. 2015, Article ID 649046, 10 pages, 2015.

[4] N. Kolarevic, M. Ognjanovic, and M. Milos, "Failures of multifunctional bulkhead caused by high gradient of temperature, pressure and speed of rotation," Engineering Failure Analysis, vol. 89, pp. 100-117, 2018.

[5] R. P. Skelton, "The energy density exhaustion method for assessing the creep-fatigue lives of specimens and components," Materials at High Temperatures, vol. 30, no. 3, pp. 183-201, 2013.

[6] K. Prasad, R. Sarkar, P. Ghosal, V. Kumar, and M. Sundararaman, "High temperature low cycle fatigue deformation behaviour of forged IN 718 superalloy turbine disc," Materials Science and Engineering A-Structural Materials Properties Microstructure and Processing, vol. 568, pp. 239-245, 2013.

[7] J. Wertz, T. Letcher, M. M. H. Shen, O. Scott-Emuakpor, T. George, and C. Cross, "An energy-based axial isothermalmechanical fatigue lifing method," Journal of Engineering for Gas Turbines and Power-Transactions of the ASME, vol. 134, no. 10, article 102502, 2012.

[8] D. Y. Hu, J. X. Mao, and R. Q. Wang, “Optimization strategy for a shrouded turbine blade using variable-complexity modeling methodology," AIAA Journal, vol. 54, no. 9, pp. 2808-2818, 2016.

[9] S. P. Zhu, H. Z. Huang, V. Ontiveros, L. P. He, and M. Modarres, "Probabilistic low cycle fatigue life prediction using an energy-based damage parameter and accounting for model uncertainty," International Journal of Damage Mechanics, vol. 21, no. 8, pp. 1128-1153, 2012.

[10] J. Fan, H. M. Liao, H. Wang et al., "Local maximum-entropy based surrogate model and its application to structural reliability analysis," Structural and Multidisciplinary Optimization, vol. 57, no. 1, pp. 373-392, 2018.

[11] Z. G. Wei, F. L. Yang, B. Lin, L. M. Luo, D. Konson, and K. Nikbin, "Deterministic and probabilistic creep-fatigueoxidation crack growth modeling," Probabilistic Engineering Mechanics, vol. 33, pp. 126-134, 2013.

[12] X. Wang, M. Rabiei, J. Hurtado, M. Modarres, and P. Hoffman, "A probabilistic-based airframe integrity management model," Reliability Engineering and System Safety, vol. 94, no. 5, pp. 932-941, 2009.

[13] S. Sankararaman, Y. Ling, and S. Mahadevan, "Uncertainty quantification and model validation of fatigue crack growth prediction," Engineering Fracture Mechanics, vol. 78, no. 7, pp. 1487-1504, 2011.

[14] M. Guida and F. Penta, "A Bayesian analysis of fatigue data," Structural Safety, vol. 32, no. 1, pp. 64-76, 2010.
[15] Y. Zhao, B. Yang, and Z. Zhai, "The framework for a strainbased fatigue reliability analysis," International Journal of Fatigue, vol. 30, no. 3, pp. 493-501, 2008.

[16] Y. Zhao, "A fatigue reliability analysis method including super long life regime," International Journal of Fatigue, vol. 35, no. 1, pp. 79-90, 2012.

[17] D. Y. Hu, Q. H. Ma, L. H. Shang, Y. Gao, and R. .Q. Wang, "Creep-fatigue behavior of turbine disc of superalloy GH720Li at $650^{\circ} \mathrm{C}$ and probabilistic creep-fatigue modeling," Materials Science and Engineering A-Structural Materials Properties Microstructure and Processing, vol. 670, pp. 17-25, 2016.

[18] S. P. Zhu, H. Z. Huang, W. W. Peng, H. K. Wang, and S. Mahadevan, "Probabilistic physics of failure-based framework for fatigue life prediction of aircraft gas turbine discs under uncertainty," Reliability Engineering and System Safety, vol. 146, pp. 1-12, 2016.

[19] S. P. Zhu, S. Foletti, and S. Berrtta, "Probabilistic framework for multiaxial LCF assessment under material variability," International Journal of Fatigue, vol. 103, pp. 371-385, 2017.

[20] R. Q. Wang, X. Liu, D. Y. Hu, F. C. Meng, D. Li, and B. Li, "Zone-based reliability analysis on fatigue life of GH720Li turbine disk concerning uncertainty quantification," Aerospace Science and Technology, vol. 70, pp. 300-309, 2017.

[21] G. R. Leverant, H. R. Millwater, R. C. McClung, and M. P. Enright, "A new tool for design and certification of aircraft turbine rotors," Journal of Engineering for Gas Turbines and Power-Transactions of the ASME, vol. 126, no. 1, pp. 155-159, 2004.

[22] Y. T. Wu, M. P. Enright, and H. R. Millwater, "Probabilistic methods for design assessment of reliability with inspection," AIAA Journal, vol. 40, no. 5, pp. 937-946, 2002.

[23] M. P. Enright, S. J. Hudak, R. C. McCung, and H. R. Millwater, "Application of probabilistic fracture mechanics to prognosis of aircraft engine components," AIAA Journal, vol. 44, no. 2, pp. 311-316, 2006.

[24] W. A. Grell and P. J. Laz, "Probabilistic fatigue life prediction using AFGROW and accounting for material variability," International Journal of Fatigue, vol. 32, no. 7, pp. 1042-1049, 2010.

[25] Z. C. Peng, H. Z. Huang, H. K. Wang, S. P. Zhu, and Z. Q. Lv, "A new approach to the investigation of load interaction effects and its application in residual fatigue life prediction," International Journal of Damage Mechanics, vol. 25, no. 5, pp. 672-690, 2016.

[26] C. Y. Zhang, L. K. Song, C. W. Fei, C. Lu, and Y. M. Xie, "Advanced multiple response surface method of sensitivity analysis for turbine blisk reliability with multi-physics coupling," Chinese Journal of Aeronautics, vol. 29, no. 4, pp. 962-971, 2016.

[27] S. Sankararaman, Y. Ling, C. Shantz, and S. Mahadevan, "Inference of equivalent initial flaw size under multiple sources of uncertainty," International Journal of Fatigue, vol. 33, no. 2, pp. 75-89, 2011.

[28] C. W. Fei, G. C. Bai, W. Z. Tang, and Y. S. Choy, "Optimum control for nonlinear dynamic radial deformation of turbine casing with time-varying LSSVM," Advances in Materials Science and Engineering, vol. 2015, Article ID 680406, 9 pages, 2015.

[29] J. X. Mao, D. Y. Hu, D. Li, R. Q. Wang, and J. Song, "Novel adaptive surrogate model based on LRPIM for probabilistic analysis of turbine disc," Aerospace Science and Technology, vol. 70, pp. 76-87, 2017. 
[30] J. Li and D. B. Xiu, "Evaluation of failure probability via surrogate models," Journal of Computational Physics, vol. 299, no. 23, pp. 8966-8980, 2010.

[31] S. Adhikari, R. Chowdhury, and M. I. Friswell, "High dimensional model representation method for fuzzy structural dynamics," Journal of Sound and Vibration, vol. 330, no. 7, pp. 1516-1529, 2011.

[32] F. Duprat and A. Sellier, "Probabilistic approach to corrosion risk due to carbonation via an adaptive response surface method," Probabilistic Engineering Mechanics, vol. 21, no. 3, pp. 207-216, 2006.

[33] C. W. Fei, G. C. Bai, and W. Z. Tang, "Probabilistic design of HPT blade-tip radial running clearance with distributed collaborative response surface method," Journal of Aerospace Engineering, vol. 28, no. 2, article 04014069, 2015.

[34] D. G. Giovanis, I. Papaioannou, and D. Straub, "Bayesian updating with subset simulation using artificial neural networks," Computer Methods in Applied Mechanics and Engineering, vol. 319, pp. 124-145, 2017.

[35] L. K. Song, J. Wen, C. W. Fei, and G. C. Bai, "Distributed collaborative probabilistic design of multi-failure structure with fluid-structure interaction using fuzzy neural network of regression," Mechanical Systems and Signal Processing, vol. 104, pp. 72-86, 2018.

[36] C. W. Fei and G. C. Bai, "Distributed collaborative probabilistic design for turbine blade-tip radial running clearance using support vector machine of regression," Mechanical Systems and Signal Processing, vol. 49, no. 1-2, pp. 196-208, 2014.

[37] R. E. Stern, J. Song, and D. B. Work, “Accelerated Monte Carlo system reliability analysis through machine learningbased surrogate models of network connectivity," Reliability Engineering and System Safety, vol. 164, pp. 1-9, 2017.

[38] I. Couckuyt, A. Forrester, D. Gorissen, F. D. Turck, and T. Dhaene, "Blind Kriging: implementation and performance analysis," Advances in Engineering Software, vol. 49, pp. 1-13, 2012.

[39] T. W. Simpson and F. Mistree, "Kriging models for global approximation in simulation-based multidisciplinary design optimization," AIAA Journal, vol. 39, no. 12, pp. 2233-2241, 2001.

[40] L. Y. Xiao, F. Qian, and W. Shao, "Multi-step wind speed forecasting based on a hybrid forecasting architecture and an improved bat algorithm," Energy Conversion and Management, vol. 143, pp. 410-430, 2017.

[41] M. Masikos, K. Demestichas, and E. Adamopoulou, "Mesoscopic forecasting of vehicular consumption using neural networks," Soft Computing, vol. 19, no. 1, pp. 145-156, 2015.

[42] L. K. Song, C. W. Fei, G. C. Bai, and L. C. Yu, "Dynamic neural network method-based improved PSO and BR algorithms for transient probabilistic analysis of flexible mechanism," Advanced Engineering Informatics, vol. 33, pp. 144-153, 2017.

[43] A. Sedki and D. Ouazar, "Hybrid particle swarm optimization and differential evolution for optimal design of water distribution systems," Advanced Engineering Informatics, vol. 26, no. 3, pp. 582-591, 2012.

[44] C. W. Fei, Y. S. Choy, D. Y. Hu, G. C. Bai, and W. Z. Tang, "Dynamic probabilistic design approach of high-pressure turbine blade-tip radial running clearance," Nonlinear Dynamics, vol. 86, no. 1, pp. 205-223, 2016.

[45] S. P. Zhu, Q. Liu, W. W. Peng, and X. C. Zhang, "Computational-experimental approaches for fatigue reliability assessment of turbine bladed disks," International
Journal of Mechanical Sciences, vol. 142-143, pp. 502-517, 2018.

[46] S. P. Zhu, Q. Liu, J. Zhou, and Z. Y. Yu, "Fatigue reliability assessment of turbine discs under multi-source uncertainties," Fatigue and Fracture of Engineering Materials and Structures, vol. 41, no. 6, pp. 1291-1305, 2018.

[47] China Zhijian Publishing House and Standards Press of China, Academic Committee of the Superalloys, China Superalloys Handbook, China Zhijian Publishing House and Standards Press of China, Beijing, China, 2012, in Chinese. 


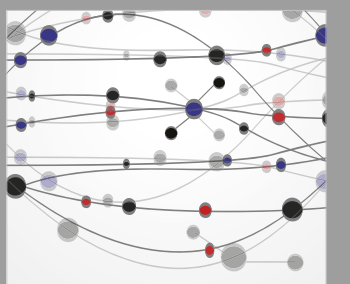

The Scientific World Journal
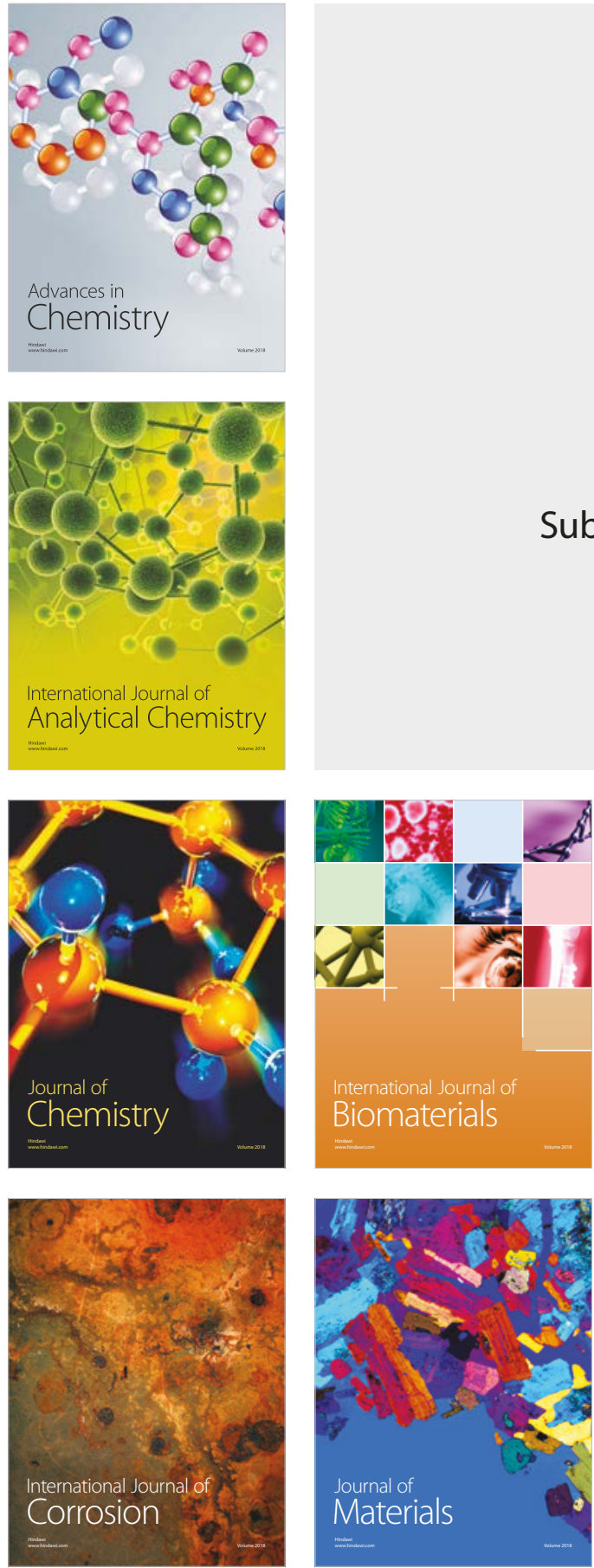

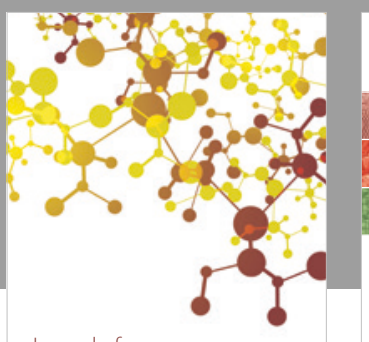

Journal of

Applied Chemistry
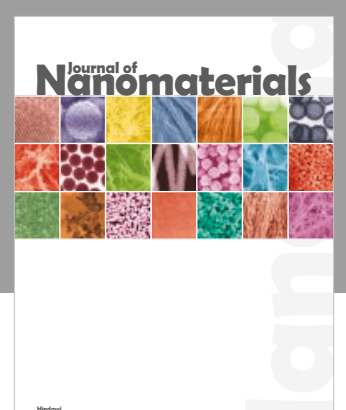

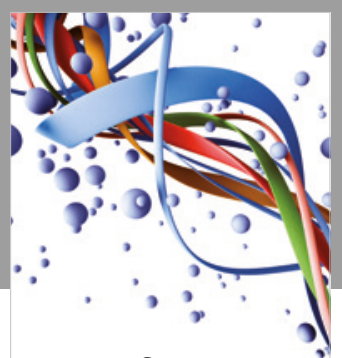

Scientifica

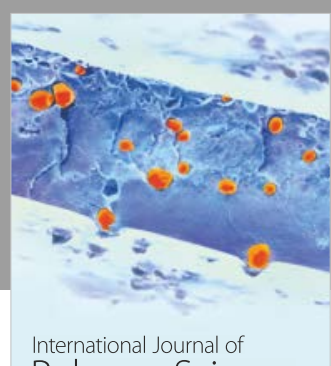

Polymer Science



Physical Chemistry
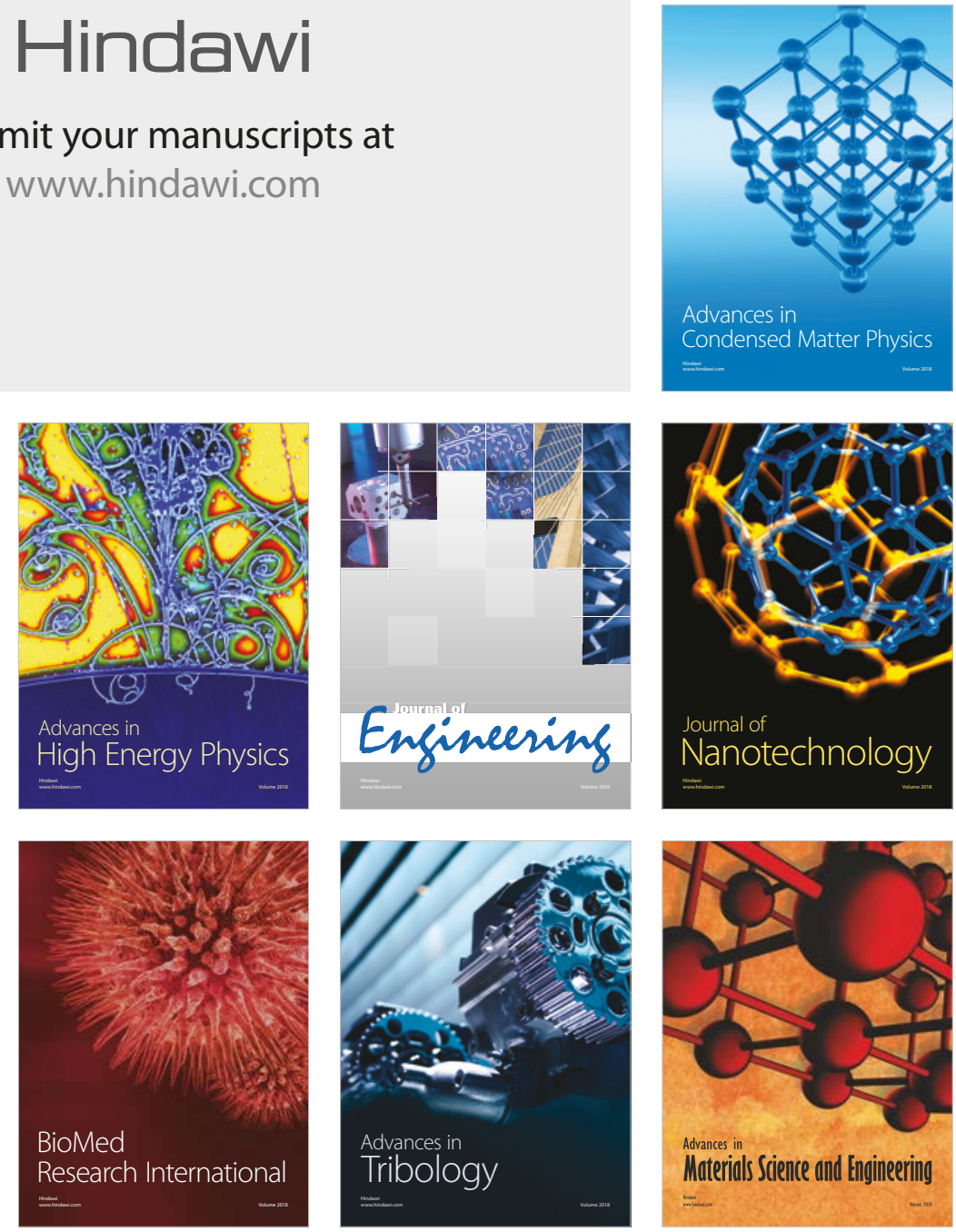